\title{
Ultrafast generation of optical and acoustic phonons in nanocrystallites
}

\author{
A. V. Bragas, ${ }^{1,2, \dagger}$ C. Aku-Leh, ${ }^{2}$ and R. Merlin ${ }^{2}$ \\ ${ }^{1}$ Departamento de Física, FCEyN, Universidad de Buenos Aires, 1428 Buenos Aires. Argentina. \\ ${ }^{2}$ FOCUS Center and Department of Physics, The University of Michigan, Ann Arbor, MI 48109-1120.USA
}

\begin{abstract}
We report on the impulsive generation of optical and acoustic phonons in $\mathrm{CdTe}_{0.68} \mathrm{Se}_{0.32}$ nanocrystallites embedded in glass, at room temperature. Using ultrafast laser pulses in a pump-probe configuration, we were able to generate coherent vibrations. The energy of our laser was tuned to the absorption edge of the nanocrystals so as to resonantly excite the quantum dots. We identified two longitudinal optical phonons, an optical mode of mixed longitudinaltransverse nature and a longitudinal-like acoustic mode. The frequency, amplitude, decay and phase as a function of excitation energy were determined for the optical modes. These results clearly identify impulsive stimulated Raman scattering as the underlying mechanism of the coherent field generation. The acoustic oscillations are associated with the lowest confined acoustic mode with pseudo angular momentum $l=0$. We find that the frequency of this mode increases as the laser central energy increases. Since the energy of the exciton at the fundamental gap depends strongly on the particle size, such a behavior is attributed to resonant size-selective excitation of the nanocrystallites. In contrast, spontaneous Raman measurements obtained from the same sample do not show size selectivity and, in addition, the resonant spectra show $l=1$ and $l=2$ modes, which are not seen in the pump-probe data. Possible explanations and comparison with other reports are discussed.
\end{abstract}

Keywords: coherent phonon, confined acoustic phonon, semiconductor quantum dot, stimulated Raman, laser pulse.

\section{INTRODUCTION}

Doped semiconductor glasses have attracted considerable attention during the last few years due to potential applications in optoelectronic devices. Since the glass matrix is transparent in the visible range, the linear and nonlinear optical properties of these composite materials are determined by those of the semiconductor nanocrystallites or quantum dots (QDs). Because the emission of phonons is one of the most important electronic dephasing mechanisms, there has been much interest in the vibrational properties of QDs. Optical phonons, ${ }^{1,2,3}$ confined acoustic phonons, ${ }^{2,4-7}$ surface phonons ${ }^{7,8}$ and disorder-activated phonons, $s^{9,10}$ in semiconductor nanocrystallites have been extensively investigated with spontaneous Raman scattering (RS). The propagation of subpicosecond laser pulses generally leads to the production of coherent phonon fields, the amplitude of which is resonantly enhanced when the laser central energy is tuned near an electronic resonance. The study of coherent phonons in semiconductor QDs, driven by ultrafast pulsed lasers, is relatively a new field. Coherent techniques allow direct time-domain observation as well as active control of vibrational modes. They also offer new possibilities for studying ultrafast phonon dynamics.

In this paper we report on the impulsive generation of optical and acoustic phonons in $\mathrm{CdTe}_{0.68} \mathrm{Se}_{0.32}$ nanocrystallites embedded in a glass matrix. We generate coherent oscillations by impulsive stimulated Raman scattering (SRS) using a standard pump-probe setup with ultrafast laser pulses tuned to the absorption edge of the sample. We study the frequency, amplitude, decay and phase of the oscillations and compare the results with predictions of the SRS model. ${ }^{11}$

The first observation of coherent acoustic phonons was reported by Krauss and Wise ${ }^{12}$ for PbS QDs. These authors noticed an intriguing discrepancy between spontaneous Raman and pump-probe results in that the Raman spectra reveal an extra mode. Ikezawa et al. ${ }^{13}$ reported similar results for PbSe QDs. We have also found striking differences between Raman and pump-probe spectra in $\mathrm{CdTe}_{0.68} \mathrm{Se}_{0.32}$. In particular, we find that the frequency of a fully-symmetric acoustic mode exhibits a strong dependence on the laser energy in the pump-probe, but not in the Raman data. The time-domain observations are ascribed to size-selectivity within the distribution of dots, an effect which, we believe, relies on doubleresonant terms which operate for pump-probe excitation but not for spontaneous RS. The observed size selectivity offers interesting possibilities for partially removing effects due to inhomogeneous broadening. 


\section{EXPERIMENTAL}

Our sample is a commercial 3mm-thick RG780 filter (Schott Glass Technologies, Inc.) made of semiconductor nanocrystallites of $\mathrm{CdTe}_{0.68} \mathrm{Se}_{0.32}$ embedded in a borosilicate matrix. ${ }^{1}$ Bulk $\mathrm{CdTe}_{1-x} \mathrm{Se}_{x}$ crystallizes in the zincblende structure for $x<0.36 .^{14,15}$ The energy bandgap $E_{g}$ versus composition exhibits bowing. At $x=0.32, E_{g}=1.4 \mathrm{eV}$. The electronic and hole spatial confinement in the dots produces a blue-shift of the gap, which can be determined by measuring the sample transmission. This is shown in Fig.1. By modeling the dielectric function of the composite and taking into account the size dispersion in the sample, the fit to the measurements gives the first excitonic transition at $E_{1}=1.68 \mathrm{eV}$ and the average radius of the sample $a=39 \AA^{.11}$

Bulk $\mathrm{CdTe}_{1-\mathrm{x}} \mathrm{Se}_{\mathrm{x}}$ shows two-mode phonon behavior leading to two, each, CdSe-like and CdTe-like longitudinal optical (LO) and transverse optical (TO) phonons. The frequencies of these phonons vary with the alloy composition. For $x=0.32$, the frequencies are, approximately, $190 \mathrm{~cm}^{-1}$ (LO) and $170 \mathrm{~cm}^{-1}$ (TO) for the CdSe-like, and $160 \mathrm{~cm}^{-1}$ (LO) and $140 \mathrm{~cm}^{-1}$ (TO) for the CdTe-like phonons. ${ }^{14}$ Additional modes attributed to clustering or disorder have been reported in bulk $\mathrm{CdTe}_{0.65} \mathrm{Se}_{0.35}{ }^{15}$

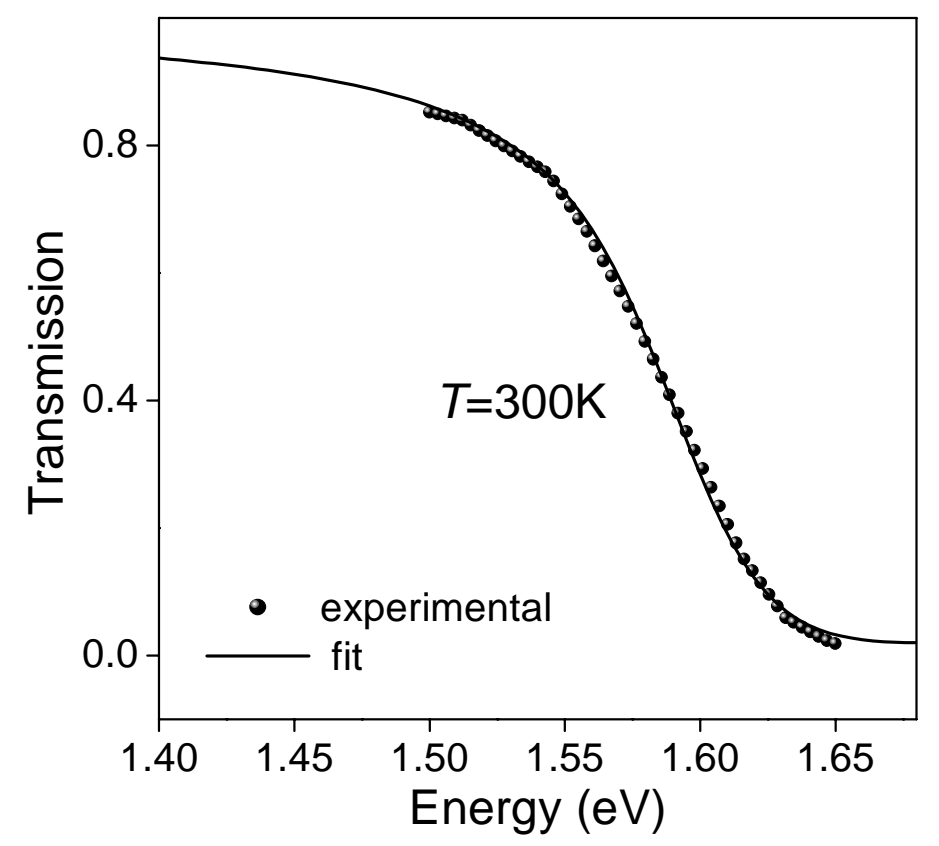

FIGURE 1: Transmission of the RG780 Schott filter near the main absorption edge.

\subsection{Pump-probe measurements}

Time-domain experiments were performed using a mode-locked Ti:Sapphire laser (Tsunami, Spectra Physics), operating at the repetition rate of $83 \mathrm{MHz}$, which emitted femtosecond pulses, tunable between 1.55 and $1.62 \mathrm{eV}$. This range covers the region of the main absorption edge of the sample; see Fig.1. The average pump intensity was set at $2 \mathrm{~kW} / \mathrm{cm}^{2}$. Special care was taken in keeping the pulse width nearly constant at about 45-50 fs.

A typical setup for pump-probe measurements is shown schematically in Fig. 2. The probe intensity was six times lower than the pump intensity. The polarizations of the pump and probe beams were perpendicular to each other to reduce the scattering of the pump into the detector. The probe transmission was measured using photodiode detectors, by balancing a portion of the probe signal before the sample with the total transmission after the sample. Lock-in detection was performed by modulating the pump intensity with a mechanical chopper at $3 \mathrm{kHz}$. 


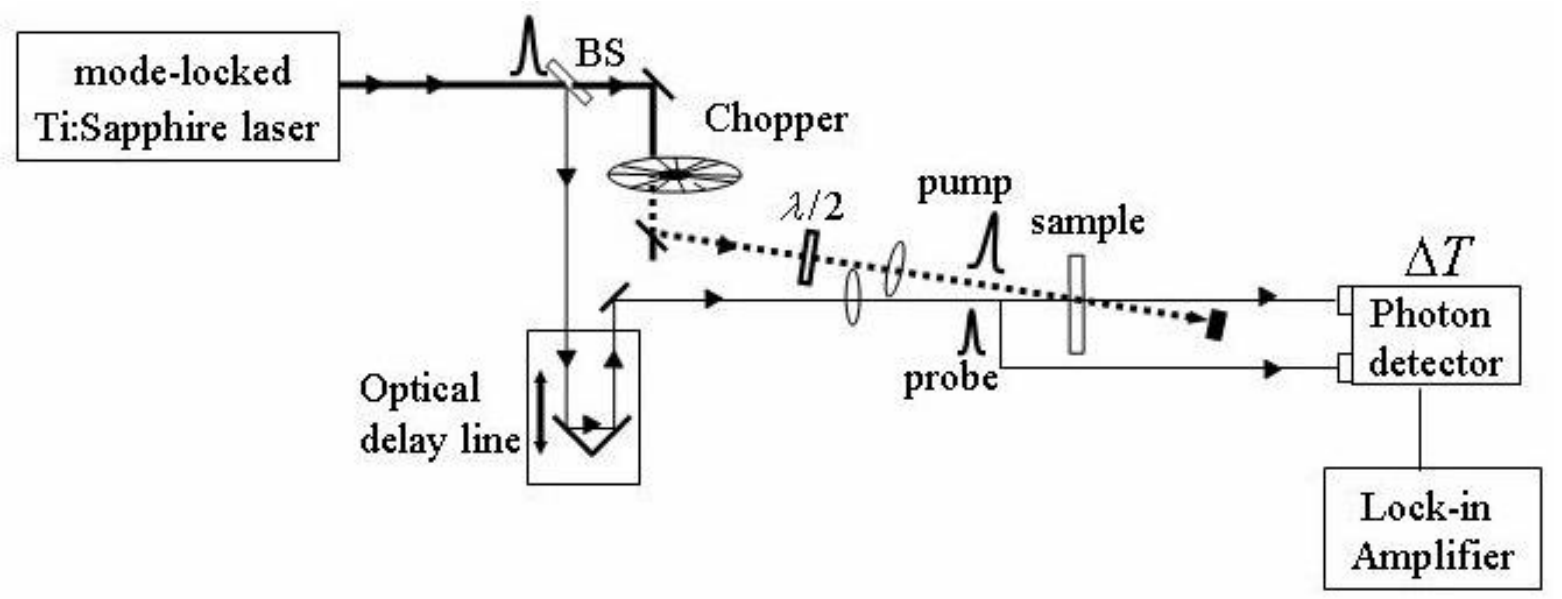

FIGURE 2: Schematics of a pump-probe experiment. BS: beam splitter, $\lambda / 2$ : half waveplate. The pump pulse is modulated with a mechanical chopper. The probe pulse goes through the optical delay line. Pump and probe beam polarizations are perpendicular to each other, to reduce the pump scattering into the detector. The total transmission of the sample is measured using a balanced photodiode detector.

\subsection{Raman measurements}

Raman measurements were performed with an XY Dilor Spectrometer. We excited the sample with a continuous wave home-made Ti:Sapphire laser, tunable between 1.53 and $1.72 \mathrm{eV}$, as well as with the $514 \mathrm{~nm}(2.41 \mathrm{eV})$ and $488 \mathrm{~nm}(2.54$ $\mathrm{eV}$ ) lines of an Argon ion laser. To avoid sample heating, we reduced the power density by focusing the light with a cylindrical lens.

\section{RESULTS AND DISCUSSION}

Figure 3 shows the normalized differential transmission, $\Delta T / T$ ( $T$ is the total probe transmission) as a function of the probe delay at the laser central energy $E_{\mathrm{c}}=1.587 \mathrm{eV}$. The raw data show oscillations due to coherent phonons superimposed on an exponential background, arising from electronic excitations. For clarity, this electronic contribution was removed from the total signal. We fit our time domain data to expressions of the form:

$$
\sum_{k} A_{k} \exp \left(-\Gamma_{k} t\right) \sin \left(\Omega_{k} t+\phi_{k}\right)
$$

using linear prediction (LP) methods. ${ }^{16}$ This procedure gives as output not only the amplitude $A$, phase $\phi$, damping $\Gamma$ and frequency $\Omega$ of each mode, but also the total number of oscillators. Fig. 3 shows an example of such a fit and, separately, the individual contributions of the various modes. Four modes can be distinguished; there are two LO modes at $\Omega=197 \mathrm{~cm}^{-1}$ and $\Omega=162 \mathrm{~cm}^{-1}$, one confined longitudinal acoustic (LA) mode at $\Omega=24 \mathrm{~cm}^{-1}$ and a fourth mode at $\Omega=140 \mathrm{~cm}^{-1}$. While the frequency of the fourth mode is close to that of the CdTe-like TO phonon, we believe that it has a mixed TO-LO character, ${ }^{17}$ as it is labeled in Fig. 3, because LO modes usually dominate the resonant spectra. We assign this mode to an optical confined mode with $l \geq 1$ where $l$ is the pseudo angular momentum. ${ }^{17} \mathrm{~A}$ detailed discussion of this assignment is given in Ref. [11]. 


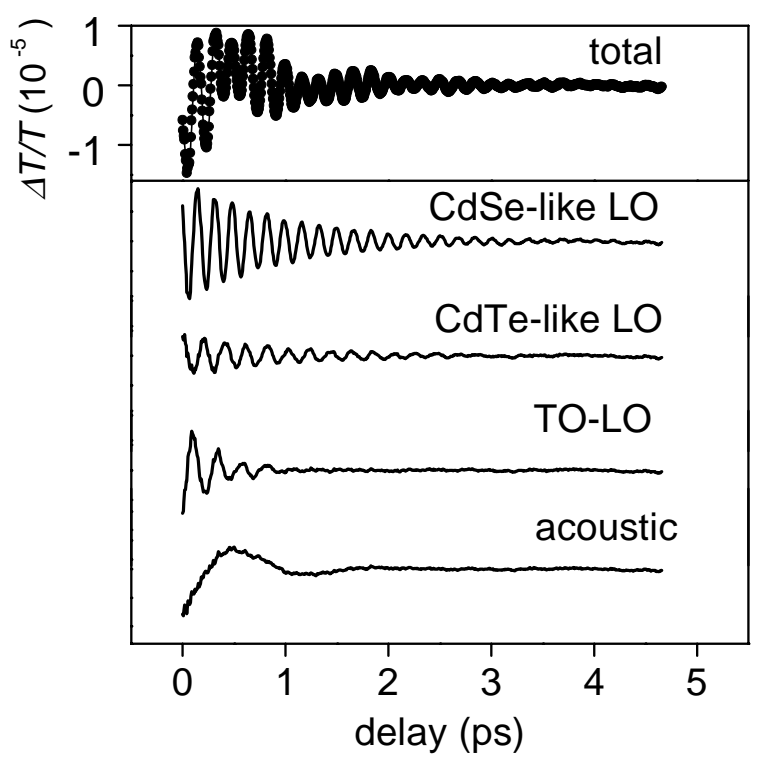

FIGURE 3: Coherent phonon signal at $E_{\mathrm{c}}=1.587 \mathrm{eV}$. The top trace shows the total normalized differential transmission, $\Delta T / T$, with the electronic background subtracted. The full circles are the experimental points. The superimposed line is the fit using LP methods. The lower panel shows the individual phonon contributions, which add up to the total signal. From top to bottom, the frequencies of the modes are: $197 \mathrm{~cm}^{-1}, 162 \mathrm{~cm}^{-1}, 140 \mathrm{~cm}^{-1}$ and $24 \mathrm{~cm}^{-1}$.

Figure 4 shows the Raman spectrum recorded in the parallel configuration (the incident and the scattered light have the same polarization). The excitation energy is $2.54 \mathrm{eV}$, well above the main absorption edge. The four modes seen in the time-domain measurements appear in the Raman spectrum, but there are two additional acoustic modes at $\sim 9 \mathrm{~cm}^{-1}$ and $\sim 15 \mathrm{~cm}^{-1}$ and several second order features above $240 \mathrm{~cm}^{-1}$. We assign the acoustic oscillations in Fig. 3, of frequency $\sim 24 \mathrm{~cm}^{-1}$, and the peak at $32 \mathrm{~cm}^{-1}$ to the same confined acoustic phonon. The reason why the frequencies in both sets of data are different is explained in Section 3.2.

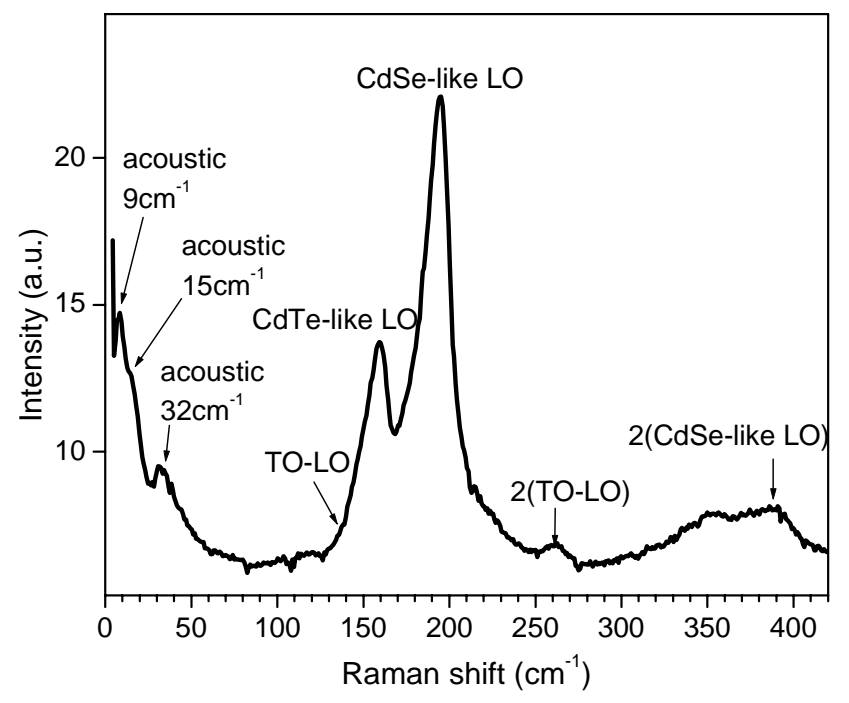

FIGURE 4: Raman spectrum at room temperature using the 488nm Ar line. 


\subsection{Optical phonons}

Stimulated Raman scattering (SRS) is determined not by one, but by two different tensors, one of which is the standard Raman susceptibility tensor, $\chi_{\mathrm{R}}$, and a second one, $\pi_{\mathrm{R}}$, which is associated with the driving force of the phonon field. ${ }^{18}$ The real parts of these two tensors are identical and, consequently, there is a single tensor for transparent materials. In this case, the light-induced driving force behaves impulsively for laser pulses that are much shorter than the phonon period. On the other hand, in absorbing regions, the imaginary components of the two tensors differ appreciably leading in most cases to an excitation-dependent displacive behavior of the phonon field. By using the proper tensor, it is possible to reproduce the impulsive behavior in the domain of transparency and the displacive behavior in the absorbing region. The complete expressions of the SRS model, as applied to nanocrystallites, are given in Ref. [11]. Here, we present some results of the model and show that the measurements unequivocally identified SRS as the underlying mechanisms for coherent phonon generation.

The $E_{\mathrm{c}}$-dependence of the coherent amplitude of the CdSe-like and CdTe-like LO modes is shown in Fig. 5. The results of SRS calculations are shown as full lines. Other than the fact that the experimental width of the resonance is slightly smaller than the calculated one, the agreement between theory and experiment is quite good. The (spectrally integrated) signal recorded in our differential transmission experiment reflects changes in the scattering spectrum. The spectrum oscillates in time with the period of the phonon and, since the sample itself acts as a variable filter, we can detect those changes as variations in the transmitted signal. It can be shown that the phonon amplitude should behave like the first derivative of the sample transmission as a function of the laser central energy, ${ }^{11}$ giving the resonant behavior shown in Fig. 5. The total signal is the sum of contributions due to all the dots, filtered by the total transmission of the sample. Our calculations assume that the electron-phonon interaction does not depend on the dot size. This parameter enters in the expression for the Raman tensor multiplying the sample transmission.

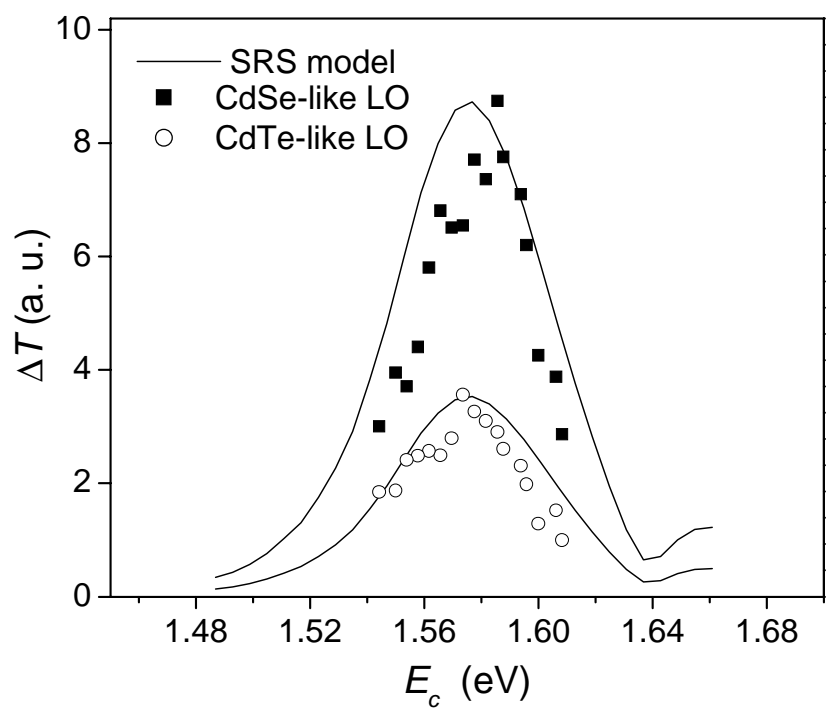

FIGURE 5: Resonant behavior of the optical phonon amplitudes and the predictions of the SRS model.

Due to a variety of sometimes competing effects, the optical frequencies are shifted with respect to bulk values. In a sample with negative phonon dispersion, confinement produces a red-shift of the phonon frequency with decreasing size and also broadens and produces asymmetric lines. ${ }^{17}$ Changes in absorption along the absorption edge can also contribute to changes in frequency of the same sign as the confinement. Another factor that introduces shifts, or more precisely blue-shifts in the LO frequency is the compressive strain, which is more pronounced for smaller dots. These effects can modify the LO frequency by $\sim 10-15 \%$ for the range from bulk to $\sim 1 \mathrm{~nm}$ QD. 
Figure 6 shows the $E_{\mathrm{c}}$ dependence of the frequencies of the optical modes. In Fig. 6(a) the CdSe-like LO frequency exhibits a small but measurable red-shift, which is consistent with the fact that smaller dots become resonantly excited when the laser energy increases. The CdTe-like LO frequency, shown in Fig.6 (b), is slightly blue-shifted. As discussed in Ref. [11], we attribute such a behavior to effects due to coupling of the CdTe-like LO phonon with the TO-LO mode, which affects the amplitude, damping, phase and frequency of both modes. ${ }^{11}$ In the next section we show that the confinement strongly affects the frequencies of the acoustic phonons. Since their dispersion is almost flat, confinement effects are not as important for optical modes.

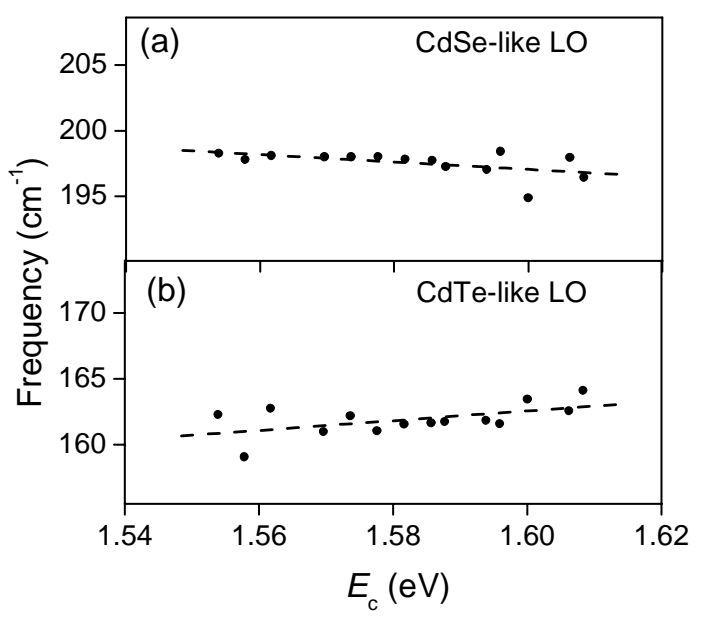

FIGURE 6: Frequency of the LO phonons as a function of $E_{\mathrm{c}}$. (a) CdSe-like LO phonon, (b) CdTe-like LO phonon. Full circles are experimental values and dashed lines are guides to the eye.

\subsection{Confined acoustic phonons}

Following the work of $\mathrm{Lamb}^{19}$ from the late $1800 \mathrm{~s}$, many authors have calculated the low frequency spectrum of nanocrystallites, by approximating them as homogeneous elastic spheres. ${ }^{4,20,21}$ In actual experiments, the assignment of acoustic modes is always challenging and often unsupported and, because of this, there is considerable confusion in the literature. Matrix properties, the value of the transverse and longitudinal sound velocity in the nanocrystallites and material inhomogeneities are some of the factors that determine the value of the acoustic frequencies and complicate the assignment. In a recent work, Saviot et al. ${ }^{22,23}$ pointed out these difficulties, giving an extensive comparison among different theoretical approaches, and showing phonon shifts and the appearance of new modes due to the matrix. On the other hand, Raman selection rules for spherical particles are well established for excitation below the gap, ${ }^{24}$ whereas forbidden acoustic as well as optical modes are often observed in the absorptive region. ${ }^{17}$

Acoustic vibrations are grouped into two categories, spheroidal and torsional. The latter involves only shear motions which are Raman inactive, ${ }^{5}$ while the former involves both shear and stretching motions. The modes of a sphere are characterized by the integer $n$ (which labels the sequence of eigenmodes in increasing order) and the spherical harmonic integers $l$ and $m(\leq l)$. The $l=0$ spheroidal mode is purely radial and fully symmetric. It has been shown that the Raman intensity for the so-called surface mode $(n=0)$ is about ten times higher than for the first overtone $(n=1) .{ }^{20}$ For transparent substances, only the $l=0$ and $l=2$ spheroidal modes are Raman active (however, see Ref. [20]). ${ }^{24}$

Independent of the boundary conditions, the acoustic phonon frequency is always proportional to the inverse radius: ${ }^{21}$

$$
\Omega_{l n}=S_{l n} \frac{v}{2 R c}
$$


Here $\Omega_{l n}$ is given in $\mathrm{cm}^{-1}$, the radius $R$ in $\mathrm{cm}$, and $v$ is the sound velocity (longitudinal for $l=0$, transversal for $l=2$ ) expressed in the same units as the speed of light, $c . S_{l, n}$ is a coefficient which depends on the ratio of the sound velocities, the choice of the boundary condition, the angular momentum $l$ and the harmonic number $n$. As the speed of sound in $\mathrm{CdTe}_{1-\mathrm{x}} \mathrm{Se}_{\mathrm{x}}$ is not known, we assume that $v_{\mathrm{L}} / v_{\mathrm{T}} \sim 2.3$, as for CdSe and CdTe. ${ }^{20}$ The coefficients $S_{l n}$ we used were gained from Ref. [21]. For fixed surface boundary conditions, $S_{00}=1.43$ and $S_{20}=2.07$. In the following we label the acoustic modes according to their frequency.

Figure 7 shows the frequency of the coherent acoustic mode as a function of the laser excitation energy. Based on the previous discussion, we assign this phonon to the fully-symmetric $\Omega_{00}$ confined mode. This assignment, and the fact that the pump-probe traces show only a single acoustic mode, is consistent with previous reports on acoustic phonon generation in rock-salt PbTe, ${ }^{25} \mathrm{PbSe}^{13}$ and $\mathrm{PbS},{ }^{12}$ zincblende $\mathrm{InAs}^{26}$ and wurzite $\mathrm{CdSe} .{ }^{26}$ Notice that, for $\mathrm{PbS}$, the $\Omega_{20}$ mode appears in the spontaneous Raman spectra, but not in the pump-probe traces. ${ }^{12}$ We further note that the differential transmission is given by $^{27}$

$$
\Delta T \sim\left(\sum_{\eta \mu} \Re_{\gamma \mu} E_{\gamma} E_{\mu}\right)\left(\sum_{\alpha \beta} \Re_{\alpha \beta} e_{\alpha} e_{\beta}\right)
$$

where $\mathfrak{R}_{\gamma \mu}$ is the Raman tensor, $\boldsymbol{E}$ is the pump field and $\boldsymbol{e}$ is the probe field. Hence, the standard pump-probe configuration we used does allow the detection of fully-symmetric modes. It is important to emphasize that the experiments reported so far were all performed under resonant conditions where the acoustic phonon signal is enhanced, as it is discussed in Section 3.1 for optical modes.

Figure 7 shows a clear size-selective excitation behavior as the laser energy is moved across the absorption edge. The selectivity manifests itself as a shift of the acoustic phonon frequency. Higher laser energies resonantly excite smaller dots inside the distribution and, consequently, higher acoustic frequencies are detected. As shown in Eq. (2), the acoustic frequency is strongly dependent on the radius of the particle. This produces substantial changes in the traces even for small changes of the laser excitation energy. Previous studies reported similar size-selective excitation in spontaneous Raman measurements on $\mathrm{CdS}^{6}$ and pump-probe measurements in PbTe. ${ }^{25}$

(a)

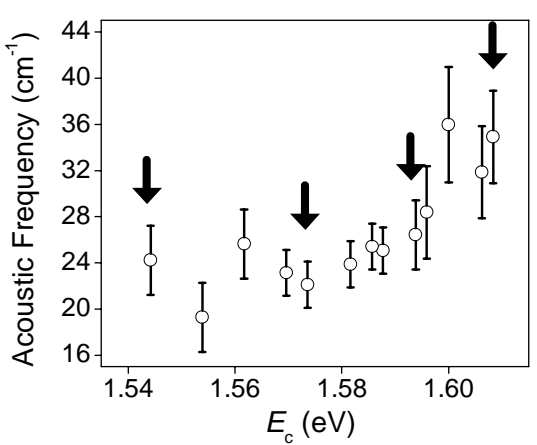

(b)

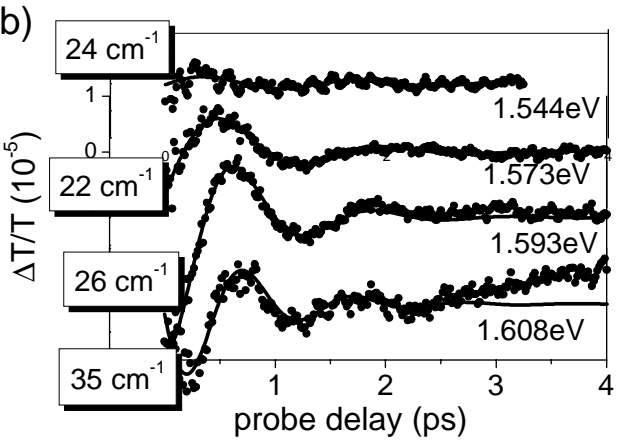

FIGURE 7: (a) Acoustic frequency as a function of the laser central energy. The frequency moves upward as the laser selectively excites smaller dots. Arrows indicate values corresponding to the four time-domain traces shown in (b).

Raman spectra were obtained in the same range of excitation energies we used in the pump-probe measurements, and also for excitation well above the first excitonic transition. Fig. 8 shows the low frequency region of the Raman spectra. In the off-resonance case we observe single broad peak, which we assign to the $\Omega_{20}$ mode. For non-resonant conditions and QDs based on cubic lattices, Montagna et al showed that the $l=2$ mode is the only one that is Raman-active. ${ }^{20}$ This is consistent with our data. The higher-frequency mode at $\sim 32 \mathrm{~cm}^{-1}$ builds up as the laser energy moves into the resonant region. This is the $\Omega_{00}$ mode which dominates the pump-probe traces. Although it is resonantly enhanced, as in the time-domain data, the $\Omega_{00}$ Raman mode does not exhibit the size-selectivity shown by the time-domain experiments. 
Rather, its frequency seems to reflect the average radius of the QD distribution. Tentatively, we attribute this difference to the fact that double-resonant terms in the Raman matrix operate for pump-probe excitation but not for spontaneous RS. $^{29}$ The reason why CdS ${ }^{6}$ but not $\mathrm{CdTe}_{0.68} \mathrm{Se}_{0.32}$ QDs exhibit size-selective RS excitation is not understood at this time. Finally, there is a third mode at $\sim 9 \mathrm{~cm}^{-1}$ which appears well-above the band gap. This peak is assigned to the Raman-forbidden $\Omega_{10}$ vibration.

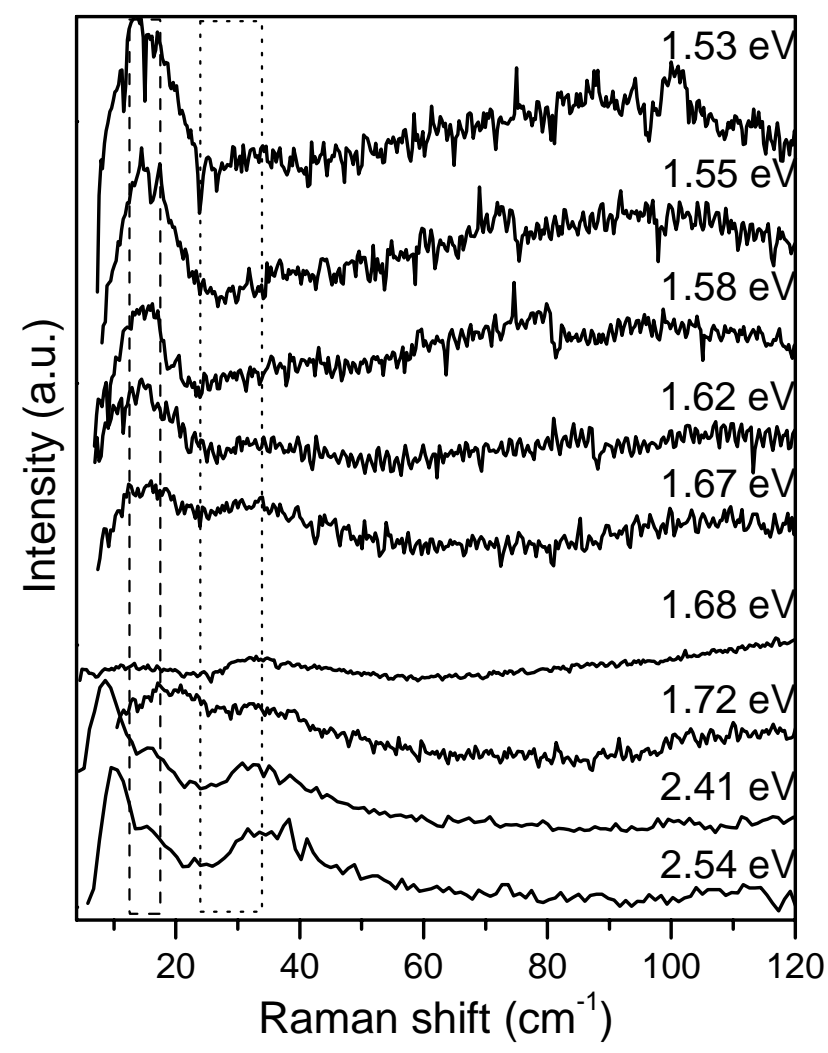

FIGURE 8: Raman spectra at $300 \mathrm{~K}$ for various laser excitation energies. The low-frequency data show three different confined acoustic phonons. The region delimited by the dotted (dashed) lines defines the range of the $\Omega_{00}\left(\Omega_{20}\right)$ acoustic mode expected for size-selective resonant excitation. The symmetric mode $\Omega_{00}$ is the peak at $\sim 33 \mathrm{~cm}^{-1}$ which exhibits a strong resonant enhancement. The only mode seen below the gap is $\Omega_{20}$. The bottom two traces, corresponding to excitation well above the gap, show an extra peak at $\sim 9 \mathrm{~cm}^{-1}$ due to the $\Omega_{11}$ mode.

Summarizing our results, Fig. 9 shows the measured dependence of the acoustic frequencies as a function of $E_{c}$. Using expressions that relate the exciton energy to the QD radius, ${ }^{28}$ the figure also shows the expected dependence on the QD radius, assuming size-selective excitation. The lines labeled $\Omega_{00}$ and $\Omega_{20}$ describe the radius dependence of the corresponding acoustic frequencies for a homogeneous distribution of QDs. Star symbols represent the Raman frequencies obtained with excitation well above the absorption edge. The measured values agree with the calculated $\Omega_{00}$, $\Omega_{20}$ and $\Omega_{10}$ frequencies for a QD radius equal to the average radius of the distribution.

The $\Omega_{00}$ frequency determined in pump-probe measurements varies in the expected manner with the resonantly selected radius, but the agreement between experiments and theory is rather poor. Consistent with size-selection, the $\Omega_{00}$ linewidth increases from $3 \mathrm{~cm}^{-1}$, at $1 / R \sim 0.2$, to $8 \mathrm{~cm}^{-1}$ for $1 / R \sim 0.22$ (not shown). ${ }^{29}$ The $\Omega_{20}$ Raman mode seems to 
follow better the theoretical predictions but, because the expected shift is rather small (see Fig. 8), it is difficult to assert as to whether or not the observed shift is due only to size-selection.

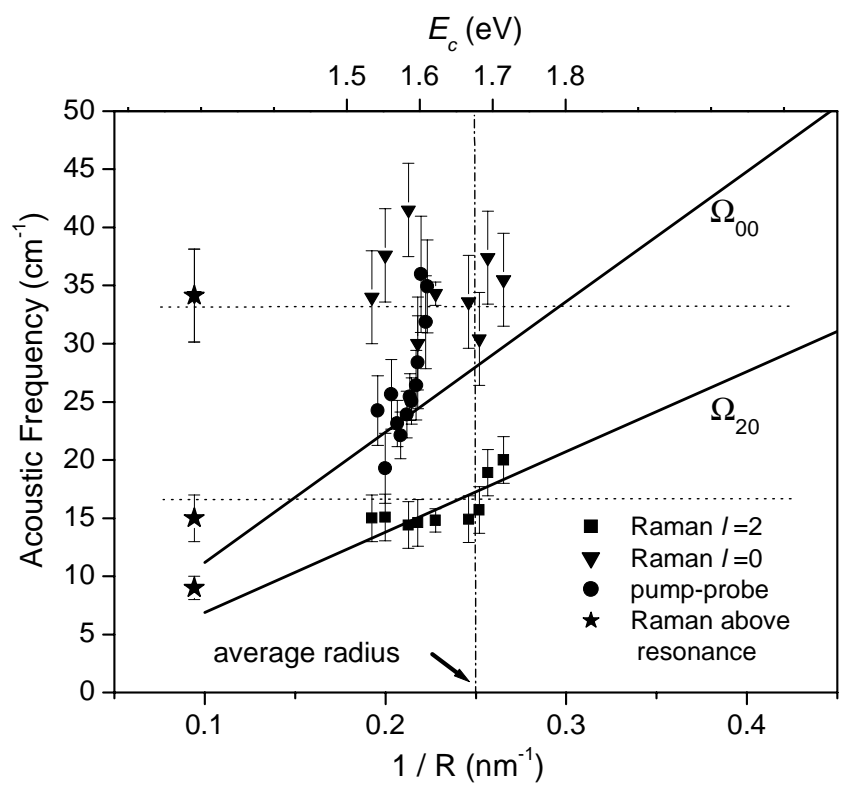

FIGURE 9: Dependence of the acoustic frequencies on $E_{c}$ and $1 / R$ (see text). Full circles correspond to pump-probe and the other symbols to RS measurements. Lines represent calculated values for a homogeneous distribution of crystallites. The strong $E_{c}$-dependence exhibited by the $\Omega_{00}$ mode (pump-probe experiments) is attributed to sizeselective excitation. RS measurements of the same mode (triangles) cluster around a value corresponding to the average radius of the distribution.

\section{CONCLUSIONS}

Four different coherent phonons have been generated in a semiconductor doped glass using femtosecond laser pulses: Two longitudinal phonons, one acoustic phonon and one confined optical mode of mixed TO-LO character. The resonant measurements for the CdSe-like and Cd-Te LO phonon support the predictions of the SRS model.

Three acoustic-related peaks were observed in spontaneous RS measurements. They were ascribed to the $\Omega_{00}, \Omega_{20}$ and $\Omega_{10}$ modes. $\Omega_{00}$ is the only mode observed in pump-probe experiments, in a geometry which favors the detection of symmetric modes. The $\Omega_{00}$ mode exhibits size-selectivity in pump-probe measurements. Its frequency increases as the laser central energy moves to higher energies. Given that the exciton energy of a nanocrystal depends on the particle size, the acoustic mode behavior is attributed to resonant size-selective excitation of the nanocrystallites, as the laser moves through the absorption edge of the sample. This selectivity is not observed in spontaneous Raman measurements even though the $\Omega_{00}$ cross section is strongly enhanced at the gap (we emphasize that this mode is not observed below the gap, consistent with the fact that the associated Raman tensor is strictly zero for cubic lattices). ${ }^{20}$

\section{ACKNOWLEDGEMENTS}

Work supported by the AFOSR under contract F49620-00-1-0328 through the MURI program. One of us (AVB) acknowledges partial support from CONICET, Argentina. 


\section{REFERENCES}

$\dagger$ e-mail: bragas@df.uba.ar

1. V. Spagnolo, G. Scamarcio, M. Lugará and G. C. Righini, Superlattices and Microstructures 16, 51 (1994).

2. T. Bischof, M. Ivanda, G. Lermann, A. Materny, W. Kiefer, J. Kalus, J. Raman Spectroscopy 27, 297 (1996).

3. W. S. O. Rodden, C. M. S. Torres, C. N. Ironside, Semicond. Sci. Tech. 10, 807 (1995).

4. P. Verma, W. Cordts, G. Irmer, and J. Monecke, Phys. Rev. B 60, 5778 (1999).

5. A. Roy, A. K. Sood, Solid State Commun. 97, 97 (1996).

6. L. Saviot, B. Champagnon, E. Duval, A. I. Ekimov, Phys. Rev. B 57, 341 (1998).

7. P. Verma, L. Gupta, S. C. Abbi, and K. P. Jain, J. Appl. Phys. 88, 4109 (2000).

8. Y. N. Hwang, S. H. Park, D. Kim, Phys. Rev. B 59, 7285 (1999).

9. A. Ingale and K. C. Rustagi, Phys. Rev. B 58, 7197 (1998).

10. M. I. Vasilevskiy, A. G. Rolo, M. J. M. Gomes, O. V. Vikhrova and C. Ricolleau, J. Phys. C: Cond. Matt. 13, 3491 (2001).

11. A. V. Bragas, C. Aku-Leh, S. Costantino, A. Ingale, J. Zhao, and R. Merlin, Phys. Rev. B 69, 205306 (2004).

12. T. D. Krauss, F. W. Wise, Phys. Rev. Lett. 79, 5102 (1997).

13. M. Ikezawa, T. Okuno, Y. Masumoto, A. A. Lipovskii, Phys. Rev. B 64, 201315 (2001).

14. Z. C. Feng, P. Becla, L. S. Kim, S. Perkowitz, Y. P. Feng, H. C. Poon, K. P. Williams and G. D. Pitt, J. Cryst. Growth 138, 239 (1994).

15. S. Perkowitz and L. S. Kim and P. Becla, Phys. Rev. B 43, 6598 (1991).

16. H. Barkhuijsen, R. De Beer, W. M. M. J. Bovée, D. Van Ormondt, J. Magn. Reson. 61, 465 (1985).

17. M. P. Chamberlain, C. Trallero-Giner, and M. Cardona, Phys. Rev. B 51, 1680 (1995).

18. T. E. Stevens, J. Kuhl and R. Merlin, Phys. Rev. B 65, 144304 (2002).

19. H. Lamb, Proc. London Math. Soc. 13, 187 (1882).

20. M. Montagna, R. Dusi, Phys. Rev. B 52, 10080 (1995).

21. L. Saviot, B. Champagnon, E. Duval, I. A. Kudriavtsev and A. I. Ekimov, J. Non-Cryst. Solids 197, 238 (1996).

22. L. Saviot, D. B. Murray and M. C. Marco de Lucas, Phys. Rev. B 69, 113402 (2004).

23. L. Saviot, D. B. Murray, Phys. Rev. Lett. 93, 055506 (2004).

24. E. Duval, Phys. Rev. B 46, 5795 (1992).

25. E. R. Thoen, G. Steinmeyer, P. Langlois, E. P. Ippen, G. E. Tudury, C. H. Brito Cruz, L. C. Barbosa, and C. L. Cesar, Appl. Phys. Lett. 73, 2149 (1998).

26. G. Cerullo, S. De Silvestri, U. Banin, Phys. Rev. B 60, 1928 (1999).

27. See, e.g., R. Merlin, Solid State Commun. 102, 207 (1997).

28. M. I. Vasilevskiı̌, E. I. Akinkina, A. M. de Paula, and E. V. Anda, Semicond. 32, 1229 (1998).

29. A. V. Bragas et al., unpublished. 